\title{
Cancer Immunotherapy: Emerging Topics and Future Perspectives
}

\author{
This article aims to present major types of cancer immunotherapy, how they are applied, and the \\ future perspectives of this field. \\ $\mathrm{Tt} \mathrm{Xu}{ }^{*}$ \\ College of Life Sciences and Medicine, Zhejiang Sci-Tech University, Hangzhou, Zhejiang 310018, China
}

\begin{abstract}
For years, cancer has been one of the most serious diseases that account for an extremely high percentage of death rate. Initially, people used chemotherapy, radiation therapy, hormonal therapy, targeted therapy, and palliative care to treat cancer. However, it was almost impossible to assure patients can live cancer-free for the rest of their life. Fortunately, with the development of immunotherapy, there have been more and more cancer patients recover from cancer successfully, which never come back within their lifetime. In this article, we talked about the major types of cancer immunotherapy, how they are applied, and the future perspectives of this field.
\end{abstract}

\section{CCS CONCEPTS}

- Applied computing; • Life and medical sciences; • Health informatics;

\section{KEYWORDS}

Adoptive cell therapy, Cytokines, Cancer vaccines, Monoclonal antibodies, Immune checkpoint inhibitors

ACM Reference Format:

$\mathrm{Tt} \mathrm{Xu}^{*}$. 2021. Cancer Immunotherapy: Emerging Topics and Future Perspectives: This article aims to present major types of cancer immunotherapy, how they are applied, and the future perspectives of this field.. In 2021 5th International Conference on Medical and Health Informatics (ICMHI 2021), May 14-16, 2021, Kyoto, Japan. ACM, New York, NY, USA, 7 pages. https://doi.org/10.1145/3472813.3473218

\section{INTRODUCTION}

Cancer is a disease that can onset in almost any organ or tissue in the body when abnormal cells grow uncontrollably. Metastatic tumors go beyond their usual boundaries to invade adjoining parts of the body and/or spread to other organs [1]. Around the world, cancer is the second leading cause of death. In 2018, there were about 10 million people died of cancer. Also, it is predicted that the cancer burden will continue to grow. According to the data from the Global Health Data Exchange (GHDx), the total number of people suffering from cancer have increased dramatically from

Permission to make digital or hard copies of all or part of this work for personal or classroom use is granted without fee provided that copies are not made or distributed for profit or commercial advantage and that copies bear this notice and the full citation on the first page. Copyrights for components of this work owned by others than the author(s) must be honored. Abstracting with credit is permitted. To copy otherwise, or republish, to post on servers or to redistribute to lists, requires prior specific permission and/or a fee. Request permissions from permissions@acm.org.

ICMHI 2021, May 14-16, 2021, Kyoto, Japan

(c) 2021 Copyright held by the owner/author(s). Publication rights licensed to ACM. ACM ISBN 978-1-4503-8984-6/21/05 ..\$15.00

https://doi.org/10.1145/3472813.3473218
1990 to 2017 [2]. In the US, every 1 in 2 men and 1 in 3 women are predicted to have cancer in their lifetime

Traditionally, we treat cancer by surgery, chemotherapy, radiation therapy, hormonal therapy, targeted therapy, and palliative care. However, it was almost impossible to truly cure patients and guarantee cancer-free for the rest of their life. Nowadays, there is a fastdeveloping type of cancer treatment: immunotherapy. Immunotherapy focuses on the immune system, which fights infections and other diseases. Immune system of human body is composed of leukocytes, organs, and tissues of the lymph system. Functions of immune system include detection and elimination of abnormal cells. As immune cells have been found in and around tumor tissues, the potential involvement of immune system in preventing and curbing the growth of cancers is most likely to happen. These identified cells, called tumor-infiltrating lymphocytes or TILs, are promising signs that the immune system is responding to the tumor.

Immunotherapy, the most promising direction of cancer treatment nowadays, has its specific advantages. Unlike traditional therapies, which act directly on cancerous tumors, immunotherapy treats patients by affecting their immune systems. By boosting the immune responses and training the immune cells to identify and destroy cancer cells, immunotherapy relies on and modifies the immune system to fight cancer. In addition, immunotherapy provides long-term protection in comparison with most conventional cancer therapies, as effectiveness of the latter would vanish once patients stop taking the drugs. This is because the immune system can not only recognize and destroy cancer cells but remember the characteristics of them. And the "memory" is what makes longer-lasting remissions possible.

In the following part of this paper, we will discuss the major types of immunotherapy: adoptive cell therapy, cytokines, cancer vaccines, monoclonal antibodies, and immune checkpoint inhibitors, as well as their future directions of development.

\section{ADOPTIVE CELL THERAPY}

Adoptive cell therapy, also known as cellular immunotherapy, is a form of treatment that uses the cells of our immune system to eliminate cancer.

There are two main approaches to adoptive cell therapy. One is by isolating the immune cells from patients and then expanding the cells in vitro, and finally, those expanded cells are reintroduced into the cancer patient. In this approach, immune cells are directly isolated and simply expanded to obtain a much larger number of them to fight cancer. The other one uses genetically modified immune cells (via gene therapy), whose cancer-fighting capabilities 

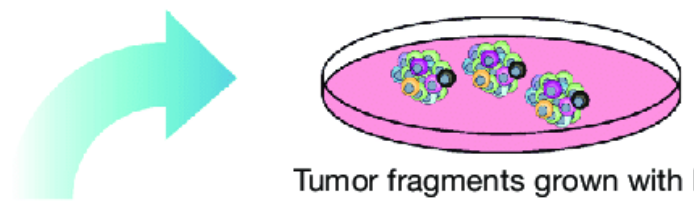

Tumor fragments grown with IL-2
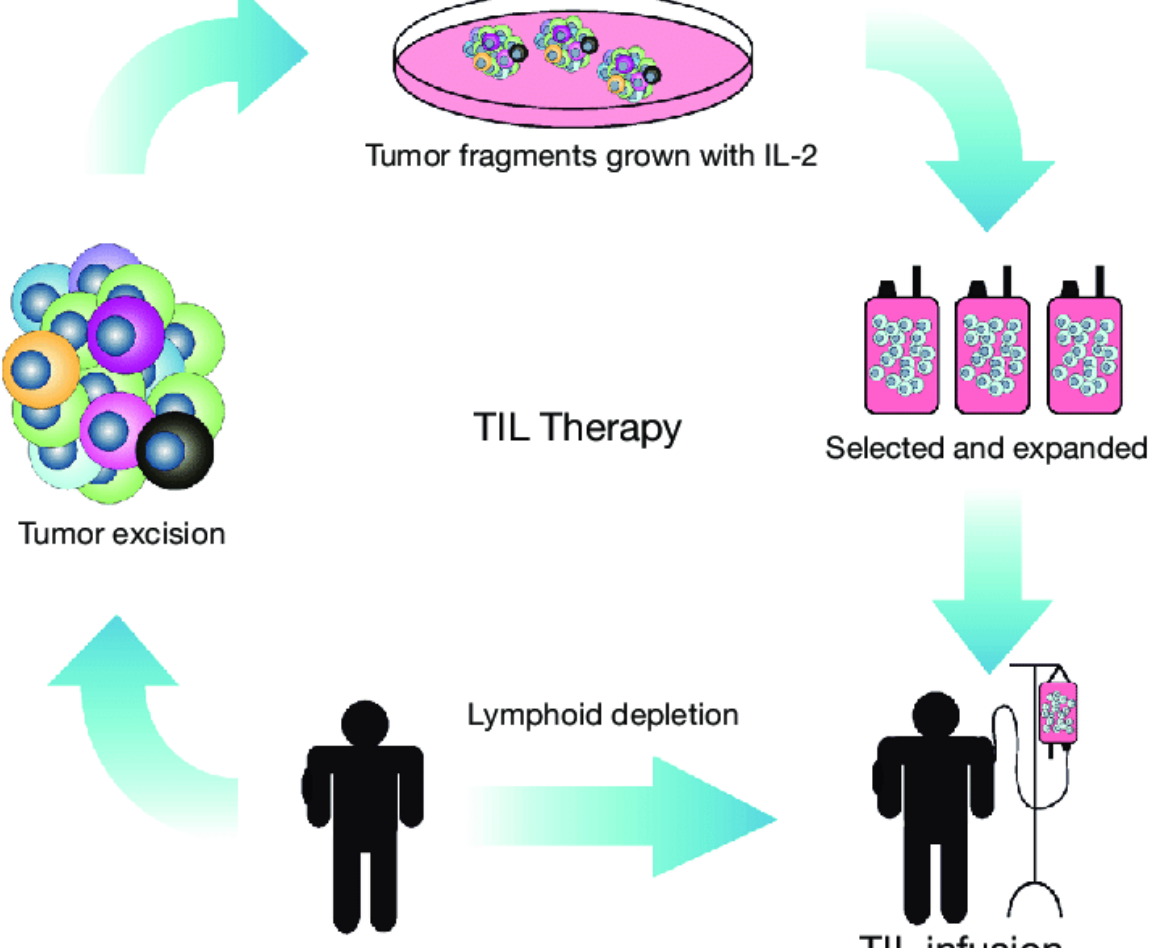

Tumor excision

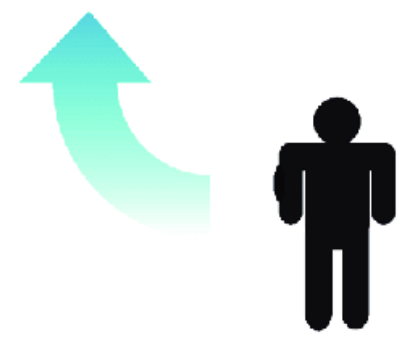

Lymphoid depletion

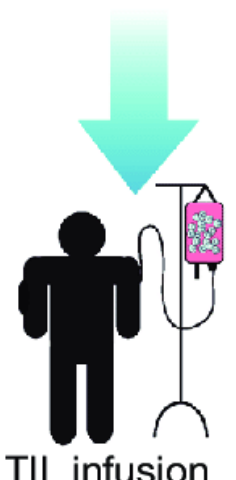

Figure 1: Flow of TIL therapy. [Public domain], via ResearchGate. (https://www.researchgate.net/figure/A-schematic-overviewof-adoptive-T-cell-transfer-or-TIL-therapy-The-tumor-is-removed_fig1_305479868)

have been boosted, and then these cells also need to be reintroduced into the cancer patient.

Adoptive cell therapy can be divided into four types: 1) tumorinfiltrating lymphocyte (TIL) therapy; 2) engineered T cell receptor (TCR) therapy, 3) chimeric antigen receptor (CAR) T cell therapy; and 4) natural killer (NK) cell therapy.

\subsection{Tumor-Infiltrating Lymphocyte (TIL) therapy}

TIL therapy uses T cells called tumor-infiltrating lymphocytes that are found in the patient's tumor. The fundamental idea behind this approach is that the lymphocytes, which are in or near the tumor, possess the ability to recognize the tumor cells. But there may not be enough of them to kill the tumor or to overcome the signals that the tumor is releasing to suppress the immune system. In this scenario, giving patients large numbers of the lymphocytes that react best with the tumor can help to overcome these barriers (Figure 1).

Initially, TIL therapy is designed for patients at an advanced stage of metastatic melanoma, and until now, metastatic melanoma is still the sole type of cancer that TIL therapy has been mostly used for, though some other kinds of cancer disease can also be treated by it, such as disseminated breast and ovarian cancer. During the period of treatment, the cytotoxicity can be significantly increased in the early stage. However, the effectiveness of the therapy would not be maintained after the end of treatment. To extend the killing effects, antigen-specific stimulated cytotoxic T-lymphocytes were used [3]. Later, there is about $50 \%$ of the patients can be effectively treated by TIL therapy and the responses have become more durable [4], [5].

The landscape of cancer treatment is being shifted by the use of checkpoint blockade and combination therapies are being evaluated. It is important to find the appropriate way of combination and sequence of TIL therapy and other therapies. Recent studies have shown that there are deleterious effects if anti-CTLA4 exposure is performed before TIL ACT responses, and to select the suitable sequence of immunotherapies, baseline IL9 levels can be used as a predictive tool [6]. Besides, the combination of TIL and anti-PD1 therapy has been proved to be safe and effective in metastatic osteosarcoma patients with chemotherapy resistance [7].

However, as has been evaluated, to be effective, this therapy needs to be administrated locally to tumors, and more clinical trials are still ongoing [8]. Besides, it needs pre-existing $\mathrm{T}$ cells that are active to tumor cells, but sometimes those T cells are not effective enough. Luckily, the latter could be handled by engineered $\mathrm{T}$ cell receptor (TCR) therapy and chimeric antigen receptor (CAR) T cell therapy.

\subsection{Engineered $\mathrm{T}$ cell receptor (TCR) therapy}

TCR Therapy is the first technological revolution in Adoptive cell therapy. As its name suggests, it uses genetic modification to change 


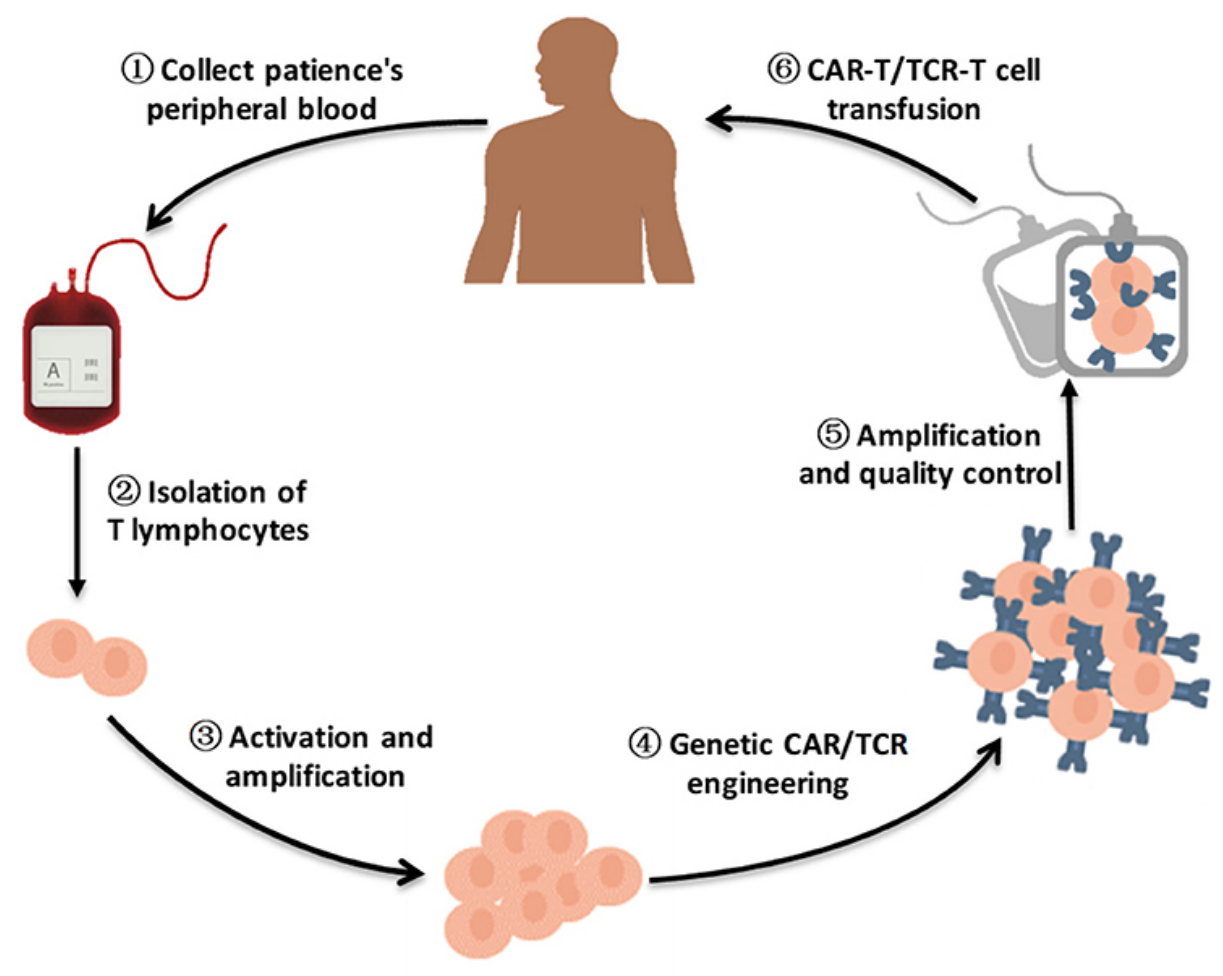

Figure 2: The flow of CAR-T and TCR-T cell therapy. [Public domain], via Frontiers in Immunology. (https://www.frontiersin. org/articles/10.3389/fimmu.2019.02250/full)

T cells to target the tumor and expand the potential of ACT (adoptive $\mathrm{T}$ cell therapy).

The first TCR used in clinical trials is one that can recognize the melanocyte differentiation antigen MART-1, and tumor regressions were observed [9]. Later, by using an affinity-enhanced TCR to target cancer/testis antigen NY-ESO-1, more robust responses were observed [10]. And TCRs were then applied in solid tumors. To expand the use of TCRs, new neoantigens are being discovered, also, the effectiveness and safety of TCRs need to be improved.

\subsection{Chimeric Antigen Receptor (CAR) T cell therapy}

Another revolution of ACT is CAR T cell therapy. It is also a kind of adoptive $\mathrm{T}$ cell therapy and an advanced version of it. CAR-T stands for chimeric antigen receptor $\mathrm{T}$ cells. These cells are modified $\mathrm{T}$ cells that can express certain kinds of fusion protein on their membrane. The fusion protein is the chimeric antigen receptor, which functions as a type of "heat-seeking missile," enabling the modified cells to produce chemicals that kill cancer (Figure 3).

CAR-T therapy is available to some children with leukemia and some adults with lymphoma. About other types of cancer, it can be a part of the treatments.

\subsection{Natural killer (NK) cell therapy}

NK cells can also be used as the material to fight cancer. As resting NK cells are activated by synergistic pairs of coactivation receptors, combining signaling domains of such receptors in NK-tailored CARs might be beneficial. And this strategy has been tested for the antitumor activity of CARs. Other novel approaches, such as the therapy relying on engineered NK-92 cells with a CAR recognizing human prostate-specific membrane antigen (PSMA) to treat prostate cancer (PCa) [11] are still under study.

\section{CYTOKINES}

Cytokines are a group of proteins in the body that plays an important part in boosting the immune system. Two types of most famous cytokine in cancer immunotherapy are interferon and interleukin (IL), respectively. Scientists have now developed man-made versions of interleukin-2 (IL-2), called aldesleukin, to treat cancer.

Interferon and aldesleukin work in several ways, including interfering with the way that cancer cells grow and multiply, stimulating the immune system and encouraging killer T cells and other cells to attack cancer cells, and encouraging cancer cells to produce chemical entities that recruit immune cells to their surrounding and attack them.

The major limitation of cytokines is the severe dose-limiting toxicities. To reduce toxicities, cytokines are being modified in two ways. For the first way, chimeric antibody-cytokine fusion proteins, also called immunokines, are emerging. For the other way, 
a
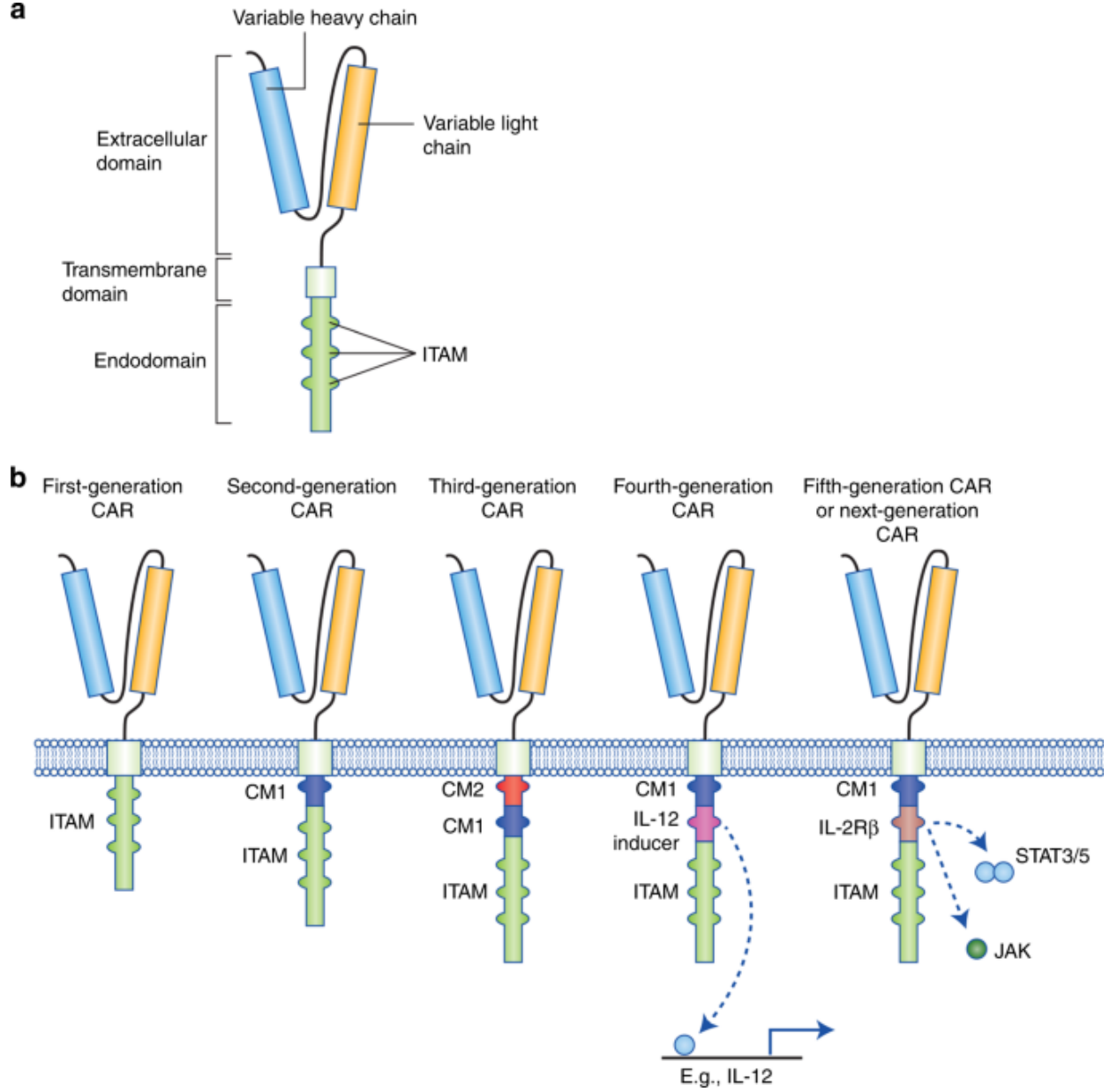

Figure 3: The structure of a CAR (a) and the five generations of CARs (b). [Public domain], via the British Journal of Cancer. (https://www.nature.com/articles/s41416-018-0325-1?WT.feed_name=subjects_cancer-immunotherapy)

superkines, the engineered cytokines mutants, are being developed (Figure 4). Superkines could alter the immunosuppressive tumor microenvironment, and deliver cell-killing agents without harming healthy cells, thus amplifying the immune system to fight cancer. They are mainly being studied to fully develop the potential of interleukins; more specifically, they were divided into three major families: IL-2, IL-4, and IL-13, which are the most studied ones.

\section{CANCER VACCINES}

Vaccines are medicines that help our immune system to fight diseases so that they can train the immune system to recognize and destroy harmful substances. Cancer vaccines are vaccines that are used to fight cancer.

There are two types of cancer vaccines: prevention vaccines and treatment vaccines. Prevention vaccines, as its name suggests, can prevent people from getting certain kinds of cancer diseases. Like vaccines for chickenpox or flu, they protect human body from viruses that can cause cancer. People have to inject the vaccine before the virus infects their bodies. Otherwise, prevention vaccines cannot defend against the development of the targeting disease. The most famous prevention vaccine is human papillomavirus (HPV) vaccine which protects against HPV. HPV can cause several kinds of cancer, and the FDA has approved HPV vaccines to prevent cervical, vaginal, and vulvar cancer. Cancer treatment vaccines, also called therapeutic vaccines, serve as types of immunotherapy. The vaccines work through boosting body's natural defenses to fight cancer. Doctors prescribe treatment vaccines to people who have already been diagnosed with cancer. Such kind of vaccines may prevent cancer from coming back, stop a tumor from growing or spreading, and destroy any cancer cells that are still in the body after other treatments have ended.

\subsection{How a cancer treatment vaccine works}

Usually, our immune system can recognize the abnormal substances as antigens and eliminate them. After that, the immune system can develop a "memory" of those antigens, which helps to respond faster and more effectively to the same antigens in the future. And cancer treatment vaccines can enhance the ability of the immune system to recognize and eliminate cancer-specific antigens, which always appear on the surface of cancer cells but not the healthy 


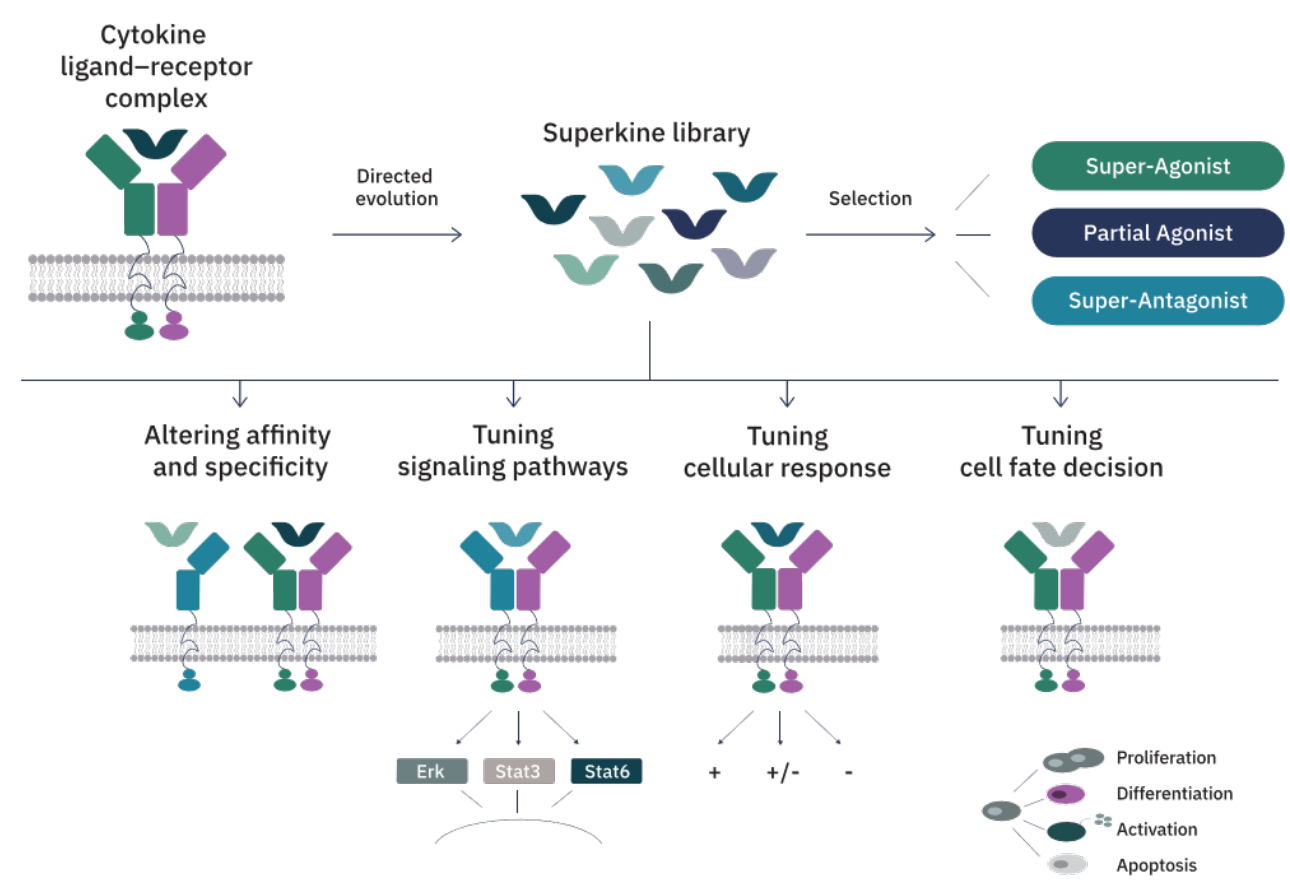

Figure 4: Directed evolution process and library of superkine. [Public domain], via Medicenna. (https://www.medicenna.com/ platform/)

cells. One way to stimulate the immune system is by giving the molecules that mimic cancer-specific antigens to the patients to recognize and destroy cancer cells that have these molecules on their surface. Also, most cancer vaccines contain adjuvants that may help strengthen the immune response. Some cancer vaccines are personalized as they are produced from the patient's tumor sample. But this kind of vaccine needs surgery operation to collect a large number of tumor cells to create the vaccine. Other types of vaccines, although targeting specific cancer antigens, are non-personalized. Because some patients have the same abnormal molecules, vaccines only have to be designed to target them.

\section{MONOCLONAL ANTIBODIES (MABS)}

Antibodies are specialized proteins made by immune system. They help human body fight against infections and disease by "recognizing" viruses, bacteria, and infected cells. Each antibody binds to a specific antigen associated with a danger signal in the body. And as monoclonal means all one type, each MAB is loads of copies of one type of antibody. As all kinds of antibodies.

MABs work by recognizing and finding specific proteins on cells (Figure 5). And each MAB recognizes one particular protein and their working ways depend on the proteins they are targeting. Some MABs trigger the immune system to attack and kill cancer cells. While others work by acting on cells of the immune system. In this way, checkpoint inhibitors also belong to monoclonal antibodies, as it can block proteins that stop the immune system from attacking cancer cells.

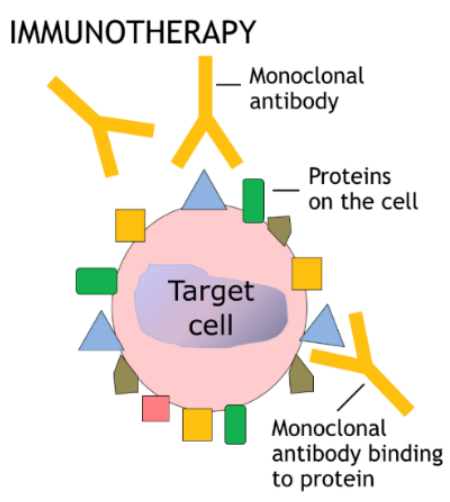

Figure 5: How a monoclonal antibody attached to a cancer cell. [Public domain], via Kleinau lab. (https://www.icm.uu. se/microbiology/kleinau-lab/)

\subsection{Naked monoclonal antibodies}

The most common kind of monoclonal antibody is the "naked" monoclonal antibody. It works on its own. Through binding to specific proteins on cancer cells, and rallying immune cells to their cause, the naked monoclonal antibodies can trigger the anti-tumor immune responses. Immune checkpoints are one of the common targets of these antibodies as they regulate the immune system. Also, other antigens working for proliferation on cancer cells can be latched onto by these antibodies. An example of a naked monoclonal antibody is trastuzumab (Herceptin), an antibody used to target the 
HER2 (human epidermal growth factor receptor 2). HER2 protein can sometimes be found on the surface of breast and stomach cells.

\subsection{Conjugated monoclonal antibodies}

The other type of monoclonal antibodies in cancer immunotherapy is conjugated monoclonal antibody. It works through carrying radioactive substances, drugs, or toxins to kill the recognized cancer cells. The treatment using antibodies with a radioactive substance belongs to radioimmunotherapy. This method can directly deliver a dose of radiation therapy to the tumor. And antibody-drug conjugates are antibodies that have chemotherapy drugs attached to them. Thus, they can deliver the toxic substance to the specific tumor cells, not healthy cells. In that way, it can eliminate the side effects caused by chemotherapy to some extent.

It is difficult to predict what the future of antibody humanization will look like. Over time, the humanization process of mouse-derived antibodies has become much more sophisticated, with several companies now performing in silico optimization of complementarity-determining regions (CDRs) for T-cell epitope avoidance to reduce immunogenicity. Yeast display is a new technology that is theoretically believed to avoid some of the disadvantages of the phage display process for monoclonal antibody generation. Newer transgenic mice that have a fuller complement of human antibody genes are also being used to develop a new generation of fully human monoclonal antibodies [12], [13].

\section{IMMUNE CHECKPOINT INHIBITORS}

There is a special type of MAB: immune checkpoint inhibitors. But because of the special and strong anti-tumor effects it shows, we classified it into another specific type. The same as all the antibodies, immune checkpoint inhibitors bind to antigens to work. But the antigens are special proteins that stop the immune system from attacking the cancer cells. In this way, inhibitors can trigger the anti-tumor response.

$\mathrm{T}$ cells have proteins on them that turn on immune response and other proteins that turn it off. These tuning proteins are called checkpoints. Some of them help T cells to become active, while others help T cells to switch off. Some cancer cells have proteins on their surface that can switch off T cells when they should be attacking the cancer cells. So, the cancer cells are pushing a stop button on the immune system. As a result, the T cells can no longer recognize and kill cancer cells.

Drugs that block checkpoint proteins are called checkpoint inhibitors. They stop the proteins on the cancer cells from pushing the stop button. This turns the immune system back on and the T cells can find and attack the cancer cells continuously.

Checkpoint inhibitors could block different kinds of checkpoint proteins including cytotoxic $\mathrm{T}$ lymphocyte-associated protein 4 (CTLA-4), programmed cell death protein 1 (PD-1), and programmed death-ligand 1 (PD-L1). CTLA-4 and PD-1 are found to be distributed on T cells while PD-L1 is on cancer cells.

Despite the clinical effectiveness of certain immune checkpoint inhibitors, the overall response rate remains low, and the effectiveness of immunotherapies for many tumors has been disappointing. There is substantial interest in looking for additional immune

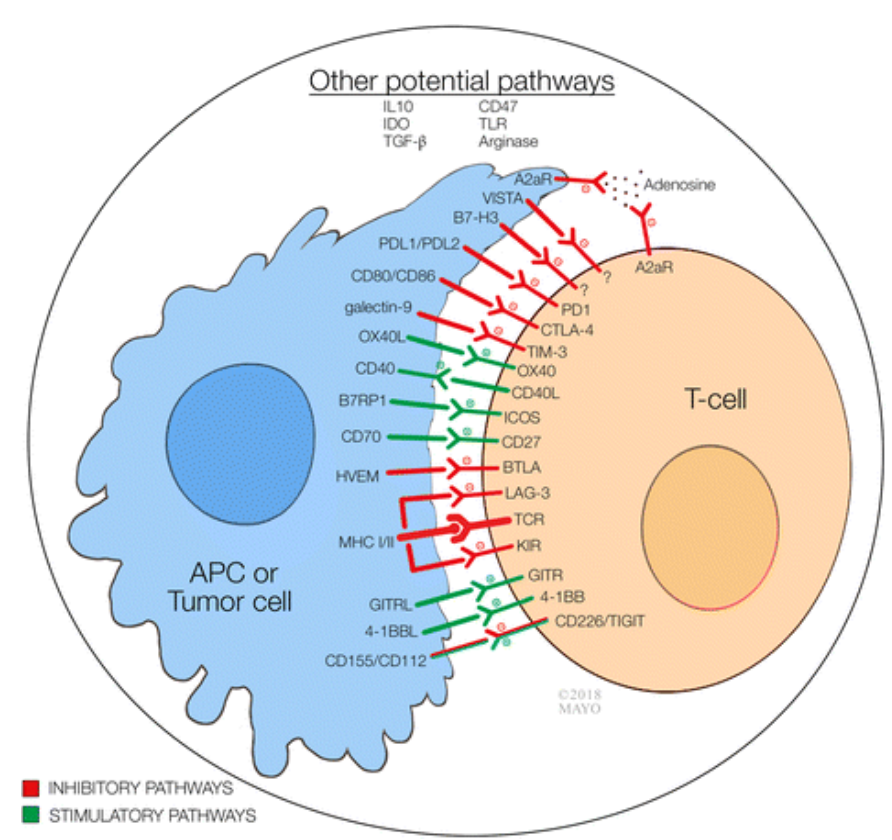

Figure 6: Future potential checkpoint pathways. [Public domain], via Journal of Hematology \& Oncology. (https: //jhoonline.biomedcentral.com/articles/10.1186/s13045-0180582-8)

checkpoint molecules that may act as therapeutic targets for cancer (Figure 6

\section{CONCLUSION}

Currently, there are mainly five types of cancer immunotherapy: adoptive $\mathrm{T}$ cell therapy, cytokines, immune checkpoint inhibitors, cancer vaccines, and monoclonal antibodies. Applications of these therapies have been observed in multiple cancer treatments.

Adoptive T cell therapy isolates and expands the immune cells in vitro and reintroduces them back into the bodies of patients to fight against cancer. Cytokines help boost the immune system, and when used in cancer treatments, it can improve anti-tumor responses. Interferon and interleukin are two major types of cytokines used as cancer immunotherapy. Immune checkpoint inhibitors trigger antitumor responses by blocking the proteins that inhibit the immune attack on the tumor. CTLA-4, PD-1, PD-L1 are the currently targeted checkpoints and new proteins are being tested. Cancer vaccines are vaccines that prevent or even fight against cancer. The most famous one is the HPV vaccine. Monoclonal antibodies used in cancer immunotherapy can recognize and find cancer-related proteins on the surfaces of cancer cells or immune cells and thus trigger antitumor responses or boost the strength of the immune system to fight against cancer. Also, MABs can carry other substances to form conjugated monoclonal antibodies, whose anti-tumor capability would thus be enlarged.

Cancer immunotherapy has shown its validity and significance in cancer treatments; in the past few years, it has been well-developed. However, there are still challenges, ranging from basic research 
to clinical development-related opportunities. For instance, the development of new preclinical models, the understanding of organspecific tumor immune context, assessing effective and efficient combinations of clinical treatments, and the continuous improvement of the regulatory endpoints for immunotherapy are what we should pay more attention to. Further, developing new delivery strategies to overcome the side effects of immunotherapy would be a promising direction. Nanoparticles, scaffolds, and hydrogels serving as ideal candidates for further application have already shown their efficiency. Though there are limitations, the bright future of immunotherapy should reasonably be expected with no doubt.

\section{REFERENCES}

[1] Rebecca L Siegel, Kimberly D Miller, and Ahmedin Jemal. 2017. Cancer Statistics, 2017. CA Cancer J Clin 67, 1 (January 2017), 7-30. https://doi.org/ $10.3322 /$ caac. 21387

[2] Max Roser and Hannah Ritchie. 2015. Cancer. Retrieved January 26, 2021 from https://ourworldindata.org/cancer

[3] Mallmann P and Krebs D. 1995. Gewinnung und Aktivierung tumorinfiltrierender Lymphozyten (TIL) im Rahmen einer Lymphokinaktivierten Killerzelltherapie (LAK-TIL-Therapie) bei Patientinnen mit fortgeschrittenen Mamma- und Ovarialkarzinom [Generation and activation of tumor-infiltrating lymphocytes (TIL) within the scope of lymphokine activated killer cell therapy (LAK-TIL therapy) in patients with advanced breast and ovarian carcinoma]. Gynakol Geburtshilfliche Rundsch 35 suppl 1 (1999), 161-165. https://doi.org/10.1159/000272590

[4] Stephanie L. Goff, Franz O. Smith, Jacob A. Klapper, Richard Sherry, John R. Wunderlich, Seth M. Steinberg, Donald White, Steven A. Rosenberg, Mark E. Dudley, and James C. Yang. 2010. Tumor infiltrating lymphocyte therapy for metastatic melanoma: analysis of tumors resected for TIL. J Immunother 33, 8 (October 2010), 840-847. https://doi.org/10.1097/CJI.0b013e3181f05b91

[5] Joost H van den Berg, Bianca Heemskerk, Nienke van Rooij, Raquel GomezEerland, Samira Michels, Maaike van Zon, Renate de Boer, Noor A M Bakker, Annelies Jorritsma-Smit, Marit M van Buuren, Pia Kvistborg, Hergen Spits, Remko Schotte, Henk Mallo, Matthias Karger, Joris A van der Hage, Michel W J M Wouters, Loes M Pronk, Marnix H Geukes Foppen, Christian U Blank, Jos H Beijnen, Bastiaan Nuijen, Ton N Schumacher and John B A G Haanen. 2020 Tumor infiltrating lymphocytes (TIL) therapy in metastatic melanoma: boosting of neoantigen-specific T cell reactivity and long-term follow-up. J Immunother Cancer 8, 2 (August 2020), e000848. https://doi.org/10.1136/jitc-2020-000848
[6] Marie-Andrée Forget, Cara Haymaker, Kenneth R Hess, Yuzhong Jeff Meng, Caitlin Creasy, Tatiana Karpinets, Orenthial J Fulbright, Jason Roszik, Scott E Woodman, Young Uk Kim, Donastas Sakellariou-Thompson, Ankit Bhatta, Arely Wahl, Esteban Flores, Shawne T Thorsen, René J Tavera, Renjith Ramachandran, Audrey M Gonzalez, Christopher L Toth, Seth Wardell, Rahmatu Mansaray, Vruti Patel, Destiny Joy Carpio, Carol Vaughn, Chantell M Farinas, Portia G Velasquez, Wen-Jen Hwu, Sapna P Patel, Michael A Davies, Adi Diab, Isabella C Glitza, Hussein Tawbi, Michael K Wong, Suzanne Cain, Merrick I Ross, Jeffrey E Lee, Jeffrey E Gershenwald, Anthony Lucci, Richard Royal, Janice N Cormier, Jennifer A Wargo, Laszlo G Radvanyi, Carlos A Torres-Cabala, Rameen Beroukhim, Patrick Hwu, Rodabe N Amaria, and Chantale Bernatchez. 2018. Prospective analysis of adoptive TIL therapy in patients with metastatic melanoma: response, impact of anti-CTLA4, and biomarkers to predict clinical outcome. Clin Cancer Res 24, 18 (September 2018), 4416-4428. https://doi.org/10.1158/1078-0432.CCR-17-3649

[7] Xiang Zhou, Junlong Wu, Chunguang Duan, and Yingjie Liu. 2020. Retrospective analysis of adoptive TIL therapy plus anti-PD1 therapy in patients with chemotherapy-resistant metastatic osteosarcoma. J Immunol Res 2020 (October 2020), 7890985. https://doi.org/10.1155/2020/7890985

[8] Y Niitsu, N Watanabe, H Kondo, and Y Kanisawa. 1990. [Receptor-mediated cancer therapy-tumoricidal cytokines, adoptive therapy of LAK, TIL]. Gan To Kagaku Ryoho 17, 6 (June 1990), 1134-1141. https://pubmed.ncbi.nlm.nih.gov/1693487/

[9] Richard A Morgan, Mark E Dudley, John R Wunderlich, Marybeth S Hughes, James C Yang, Richard M Sherry, Richard E Royal, Suzanne L Topalian, Udai S Kammula, Nicholas P Restifo, Zhili Zheng, Azam Nahvi, Christiaan R de Vries, Linda J Rogers-Freezer, Sharon A Mavroukakis, and Steven A Rosenberg. 2006. Cancer regression in patients after transfer of genetically engineered lymphocytes. Science 314, 5796 (October 2006), 126-129. https://doi.org/10.1126/science. 1129003

[10] Paul F Robbins, Sadik H Kassim, Thai L N Tran, Jessica S Crystal, Richard A Morgan, Steven A Feldman, James C Yang, Mark E Dudley, John R Wunderlich, Richard M Sherry, Udai S Kammula, Marybeth S Hughes, Nicholas P Restifo, Mark Raffeld, Chyi-Chia R Lee, Yong F Li, Mona El-Gamil, and Steven A Rosenberg. 2015. A pilot trial using lymphocytes genetically engineered with an NY-ESO-1-reactive T-cell receptor: long-term follow-up and correlates with response. Clin Cancer Res 21, 5 (March 2015), 1019-1027. https://doi.org/10.1158/1078-0432.CCR-14-2708

[11] Isabella Monia Montagner, Alessandro Penna, Giulio Fracasso, Debora Carpanese, Anna Dalla Pietà, Vito Barbieri, Gaia Zuccolotto, and Antonio Rosato. 2020. AntiPSMA CAR-engineered NK-92 cells: an off-the-shelf cell therapy for prostate cancer. Cells 9, 6 (June 2020), 1382. https://doi.org/10.3390/cells9061382

[12] Nicholas A P S Buss, Simon J Henderson, Mary McFarlane, Jacintha M Shenton, and Lolke de Haan. 2012. Monoclonal antibody therapeutics: history and future. Curr Opin Pharmacol 12, 5 (October 2012), 615-622. https://doi.org/ 10.1016/j.coph.2012.08.001

[13] Nuventra 2018. Monoclonal antibodies: past, present, and future. Retrieved January 26, 2021 from https://www.nuventra.com/resources/blog/monoclonalantibodies-past-present-and-future/ 